\title{
X-RAY SCANNING APPARATUS FOR MAPPING TEXTURE AND ELEMENT DISTRIBUTIONS
}

\author{
R. A. SCHWARZER and M. WEHRHAHN \\ Institut für Metallkunde und Metallphysik, Technische Universität Clausthal, \\ Grosser Bruch 23, D-38678 Clausthal-Zellerfeld, Germany
}

(Received 21 June 1995)

\begin{abstract}
The past decade has seen some remarkable progress in spatially resolved texture analysis due to new computer-aided techniques of electron diffraction with the SEM and TEM. To avoid, however, some limitations specific for electron microscopy, an x-ray scanning apparatus has been developed for the mapping of texture and element distributions on bulk samples. The set-up consists of a "white" $x$-ray source, a collimator system to produce a fine primary beam spot, an $x$-y sample stage operated by stepper motors, and an EDX detecting system for peak separation.

Energy dispersive $x$-ray diffraction and $x$-ray fluorescence analysis are used for data acquisition. The density distributions of selected crystallographic directions or of element concentrations in the sample surface are acquired spot by spot, and represented by pseudocolour or grey shade maps. Several texture distribution as well as element composition maps can be obtained simultaneously. Spatial resolution is presently limited to $50 \mu \mathrm{m}$ by the low level of primary beam intensity.
\end{abstract}

KEY WORDS: Local texture, energy dispersive $x$-ray diffraction, $x$-ray micro-fluorescence analysis, individual grain orientation measurement.

\section{INTRODUCTION}

The past decade has seen some remarkable progress in spatially resolved texture analysis. The modern techniques of microscale texture measurement are based on electron diffraction in the scanning (SEM) and transmission (TEM) electron microscope (Schwarzer, 1991a; Weiland, 1994). The benefits of analytical electron microscopy are the correlation of morphology (from the micrograph) and element composition (from EDX analysis) with crystal lattice orientation on a submicron scale. As an extension of $x$-ray texture analysis, quantitative pole figures can be measured by TEM in selected area diffraction mode to finally obtain the ODF for cubic crystals (Xia, Schwarzer, 1994) or texture estimates for hexagonal crystals (Helming, Schwarzer, 1994) from sample regions down to about $1 \mu \mathrm{m}$ wide. If the spatial arrangement of grains or misorientations across grain boundaries are important, individual lattice orientations have to be analysed grain by grain. They are most conveniently determined by interpreting Kikuchi patterns in reflection from bulk samples (SEM) or in transmission from transparent samples (TEM). Measurement of single grain orientations is straight forward using a computer-aided technique: The diffraction pattern on a luminescent screen in the microscope is transferred to a computer via a low light level video camera, and interpreted interactively or automatically within seconds.

The majority of individual grain orientation measurements have so far been performed by the backscatter Kikuchi pattern technique (Dingley, Randle, 1992) with the SEM (also known as electron back-scatter diffraction technique; "BKP". "EBS", "EBSP" and similar acronyms are used in literature). This is due to specific advantages of SEM 
over TEM. First of all, SEM are widely used in many laboratories and in addition, several commercial systems and computer programs for backscatter Kikuchi pattern analysis are available. The operation of an SEM is less sophisticated. The preparation of samples is extremely simple. Finally, dynamic heating or tensile stage experiments can be performed conveniently in the SEM. A fully automated orientation mapping appliance for the SEM has been developed recently which involves interfacing control of the specimen stage, pattern interpretation routines and image mapping software ("orientation image microscopy", OIM"M) (Mason, Adams, 1994). The piezoelectric driven stage has the capability of taking $0.1 \mu \mathrm{m}$ steps over approximate $27 \mathrm{~mm}$. $26 \mathrm{~mm}$ area. The reproducibility over this range of travel is $0.25 \mu \mathrm{m}$. As an alternative (Schwarzer, Kunze, 1994) the primary beam rather than the sample position is controlled by the computer, and the beam spot is positioned on the sample surface in incremental steps on a square grid pattern. In the latter version, lateral resolution and reproducibility are only limited by the spot size and the digitisation depth of the digital-to-analog converter of the beam control.

The first choice in materials science, however, might be the TEM. It enables the deepest insight in the microstructure: in addition to precise crystal lattice orientations and misorientations, the density, arrangement and type of dislocations, deformation systems, as well as the complete orientation of grain boundaries can be determined on-line (Schwarzer, Zaefferer, 1994). The spot size of a modern TEM in microbeam diffraction mode can be reduced to some $10 \mathrm{~nm}$ diameter while the Kikuchi diffraction pattern is still sufficiently bright to enable pattern recognition. Hence spatial resolution and the degree of plastic sample deformation make virtually no limitation. If an appropriate sample preparation is applied and a medium voltage microscope is available, contiguous sample areas more than $0.1 \mathrm{~mm} \cdot 0.1 \mathrm{~mm}$ wide are often electron transparent and can be studied. This means that with fine grained materials - which are the real domain of electron microscopy investigations - a large number of single grain orientations, indeed, can be measured per sample so that they are statistically sufficient for texture analysis.

When studying technical materials, however, electron diffraction suffers from several deficiencies. Rather diffuse Kikuchi patterns are formed unless the volume of the grain illuminated by the primary beam is a fairly perfect crystal. In the SEM sharp backscatter Kikuchi patterns from bulks can hardly be generated neither on submicron grained nor extensively cold deformed materials, since spatial resolution is in the range of 0.2 to $1 \mu \mathrm{m}$, depending on the beam voltage and the mean atomic number. Low electric conductivity may give rise to excessive sample charging. A thin conductive coating, however, is often prohibitive as any foreign surface layer tends to obscure the backscatter Kikuchi pattern. Furthermore the sample must be steeply tilted to the primary beam. Hence the surface image is foreshortened and distorted, and standard electron detectors often give poor image quality only. The application of the TEM, on the other hand, is handicapped by the tedious sample preparation. TEM is not adequate for coarse grain sizes. Finally there are only a few TEM to date equipped with a facility for the online measurement of SAD pole figures or individual grain orientations.

\section{EXPERIMENTAL SET-UP OF THE X-RAY SCANNING APPARATUS}

Electron microscopy cannot be used to perform all local texture measurements equally well. In spite of latest instrumental developments, there are still some applications where 
individual grain orientations cannot be measured by electron microscopy such as in the case of excessive deformation, small grain size, or incompatibility of the sample with electron microscopy. Some other applications only require a knowledge of the spatial distribution of certain crystal lattice directions averaged over small areas (i.e pole densities) in the sample surface rather than the full crystal lattice orientation of each grain.

In order to avoid some limitations of electron microscopy, an x-ray scanning technique has been developed which is based on energy dispersive $\mathrm{x}$-ray spectroscopy (Schwarzer, 1989). The spatial distributions of pole densities or elements in the sample surface are illustrated graphically by the construction of maps. Pseudocolours specific for the density of a crystallographic direction or concentration of an element under consideration are assigned to the image points $(x, y)$. Inhomogeneities in texture or composition are so easily recognised. The application of the $\mathrm{x}$-ray scanning apparatus is not limited to metal samples. Insulating polycrystals can be studied as well, such as ceramics, minerals or bones. Sample preparation is simple. In most cases the same or similar methods as for reflected light microscopy can be applied. X-ray mapping is a very gentle method, since the specimen is only exposed to $\mathrm{x}$-rays and stays in the atmospheric environment.

The preliminary set-up (Schwarzer, 1991b; Schwarzer, 1993) has been further improved. The present apparatus (Figure 1) consists of a PHILIPS generator PW 1830/

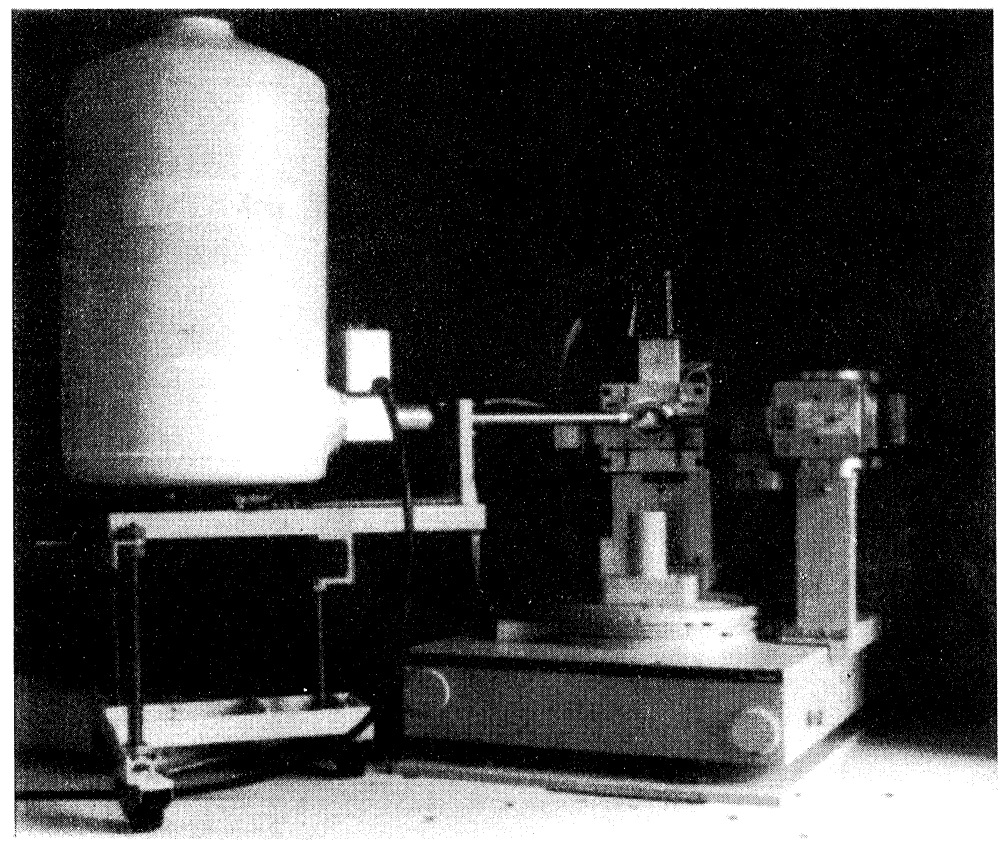

Figure 1 Experimental set-up of the x-ray scanning apparatus. 
25 equipped with a fine-focus tube and a goniometer PW 1835. A circular diaphragm collimator is attached to the tube in order to confine the beam to a fine spot. The specimen is mounted in vertical position on an $x-y$ stage with stepper motor drives (HUBER stage 5102 and control unit 9012). The stage is capable of taking $2.5 \mu \mathrm{m}$ steps over approximately $24 \mathrm{~mm} \cdot 24 \mathrm{~mm}$ sample area. The diffracted intensity values are detected with an EDX system (SPECTRACE 6100 and TRACOR NORAN Si(Li) detector). The angle of incidence, $\theta_{1}$, and the takeoff angle, $\theta_{2}$, can be varied independently between $20^{\circ}$ and $65^{\circ}$ by rotating the sample stage and the detector arm. The apparatus is controlled by a personal computer.

\section{PRINCIPLES OF ENERGY DISPERSIVE X-RAY DIFFRACTION}

The ordinary tube has a tungsten target. The high density material gives rise to an intense "white" radiation primary beam consisting of continuous Bremsstrahlung and characteristic tungsten lines. From the illuminated spot on the sample surface a wide spectrum of secondary $x$-rays is emitted which is composed of broad diffraction peaks, sharp characteristic fluorescence lines, and a.low background of scatter radiation. A crystallite diffracts partial rays from the primary beam which are matching Bragg's law (Figure 2):

$$
\mathrm{n} \lambda=2 \mathrm{~d}_{\mathrm{hkl}} \sin \theta
$$

or expressed in photon energy:

$$
\mathrm{E}_{\mathrm{hkl}}=\mathrm{h} \mathrm{c} / \lambda=0.62 \mathrm{n} /\left(\mathrm{d}_{\mathrm{hkl}} \sin \theta\right) \mathrm{nm} \cdot \mathrm{keV}
$$

$n$ stands for the order of diffraction, $\lambda$ for the $x$-ray wavelength, $d_{\text {hit }}$ for the lattice spacing, $\theta=\left(\theta_{1}+\theta_{2}\right) / 2$ for Bragg's angle, h for Planck's constant, and c for the velocity

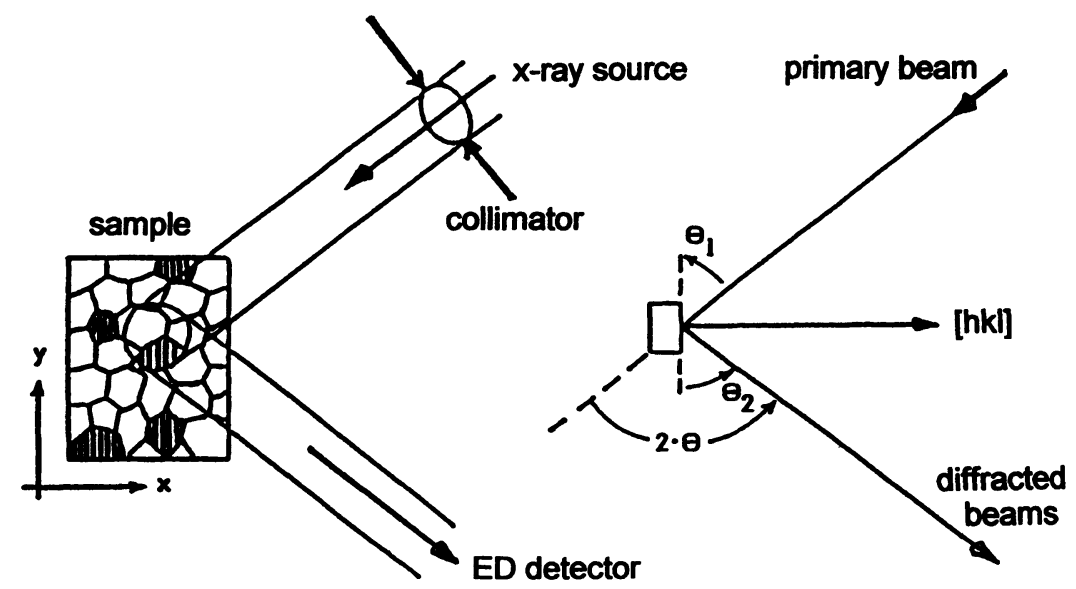

Figure 2 Schematics of an $x$-ray scanning apparatus using energy dispersive diffraction for texture mapping. 
of light. Peak separation is performed by energy dispersive $x$-ray spectroscopy. The detector measures the intensity at a given pole figure point for several diffracting lattice planes \{hkl\} simultaneously which all fulfil equation (2). Standard EDX systems such as used for materials analysis in the SEM are well suited. The energy resolution is in the range from $130 \mathrm{eV}$ to $160 \mathrm{eV}$ and sufficient for texture measurement, since diffraction peaks of low indexed planes of cubic materials are separated by several hundred electron volts in the spectrum, if the goniometer angles $\theta_{1}$ and $\theta_{2}$ have been set appropriately. In order to increase sensitivity by enhancing intensity, diffraction peaks may be positioned on the energy values of characteristic lines in the primary beam by varying $\theta_{1}$ and $\theta_{2}$ simultaneously.

The sample is moved step by step in a regular grid pattern with the $x-y$ stage, and a spectrum is acquired at each grid position. Information can be simple integrated peak intensities or processed data, such as intensities corrected for background or overlap. 25 peaks of a spectrum can be analysed simultaneously and composed to texture maps of the same sample area. The computer notes the position of the sample stage, and stores the intensity values along with the corresponding $x-y$ coordinates in memory. Once this cycle is completed, the computer increments the stage to its next measurement location, and a new spectrum is collected. For quantitative measurements, a dwelling time of typically several seconds is required for the acquisition of an image point, while a qualitative texture analysis is possible at scan rates of a second per point. The long time of data collection restricts the total number of points in a map.

As an alternative to a diffraction peak, an $\mathrm{x}$-ray fluorescence peak can be processed in the same way which is then characteristic for the concentration of the corresponding element. An element distribution map is thus obtained. The beam apertures and angular settings have no effect on $x$-ray fluorescence analysis. Absorption in air must be considered for quantum energies $<3 \mathrm{keV}$ which hinders light elements below potassium to be detected. Dedicated imaging micro-fluorescence instruments have been developed (Pella, Feng, 1992; Larsson, Engström, 1992). They are, however, not capable of texture mapping, because the settings of the angles of incidence, $\theta_{1}$, and acceptance, $\theta_{2}$, are fixed.

It should be noted that the texture maps of one run have the same sample direction in common. So energy dispersive diffraction would correspond to a $\theta / 2 \theta$ scan in a conventional Bragg-Brentano geometry, and a sequential selection of the peaks of interest at each sample point of the map. An x-ray scanning apparatus based on the BraggBrentano geometry, however, is difficult to realise. A sophisticated mechanical design is required, because the $\theta / 2 \theta$ scan has to be performed eucentricly such that the beams pivot about the measured point without translation on the sample. As an alternative the texture maps for different $\{h k l\}$, i.e. for different $\theta / 2 \theta$ settings, might be measured one after the other. In any way a sequential procedure would drastically increase the total time required for measurement. A further disadvantage of a Bragg-Brentano geometry is the steep increase of spot size with shallow incidence of the primary beam.

\section{APPLICATIONS}

In the examples shown in this study, the intensities were calculated from the peak integrals with correction for background. The texture maps were acquired at symmetrical diffraction settings $\left(\theta_{1}=\theta_{2}\right)$, i.e. the pole density distributions were probed in the sample normal direction. If the pole density distributtions are important in another direction, 
the sample has to be rotated or tilted from the symmetrical to an oblique diffraction position.

The spatial resolution of the $\mathrm{x}$-ray scanning apparatus is primarily dependent on the $\mathrm{x}$-ray spot width on the sample. Since no conventional lenses are available for white $\mathrm{x}$-rays, a beam collimator is used which consists of two small circular diaphragms. The spot size cannot be reduced indefinitely by stopping down the beam, since the intensity falls off proportionally to the spot area. A marked improvement in resolution and spot brightness is expected from the replacement of the diaphragm collimator by a glass capillary collimator (Carpenter et al, 1989; Ringby et al. 1989). Resolution further decreases due to the oblique incidence of the primary beam which results in an elliptical foreshortening of the spot on the sample surface by $1 / \sin \theta_{1}$.

\subsection{Element mapping by $x$-ray micro-fluorescence analysis}

Spatial resolution was tested by collecting an x-ray fluorescence map (Au $\mathrm{L}_{\alpha}$ lines) of a regular gold pattern (Figure 3 ). The gold patches $(420 \mu \mathrm{m} \cdot 420 \mu \mathrm{m}$ wide) were deposited on a glass substrate by sputter-coating through a TEM specimen grid. The free bars $(<60 \mu \mathrm{m}$ wide gaps) between the patches are clearly resolved in two perpendicular directions by which a spatial resolution of approximately $50 \mu \mathrm{m}$ is demonstrated.

\subsection{Texture mapping by energy dispersive $x$-ray diffraction}

The type of information displayed in diffraction maps depends on the ratio of $\mathrm{x}$-ray spot over grain size. If grain size exceeds spot size, diffraction takes place in individual grains and orientation contrast maps are obtained. Otherwise diffracted intensity is integrated over several grains and texture maps are formed. The apertures, $\Delta \theta$, of the primary and secondary beams are not critical, since texture width is in the range of several degrees in most materials.

As an example for texture mapping a longitudinal section through a rolled copper profile is shown in Figure 4. The profile was formed by pass rolling from a square to an elliptical cross-section. A section was cut through its mid-plane and a polished flat of $14 \mathrm{~mm} \cdot 19 \mathrm{~mm}$ in size was prepared for this measurement. The 220 map (Figure 4.a) was taken at slightly asymmetrical angular settings $\left(\theta_{1}=52^{\circ}, \theta_{2}=60^{\circ}, \theta=57^{\circ}\right.$,
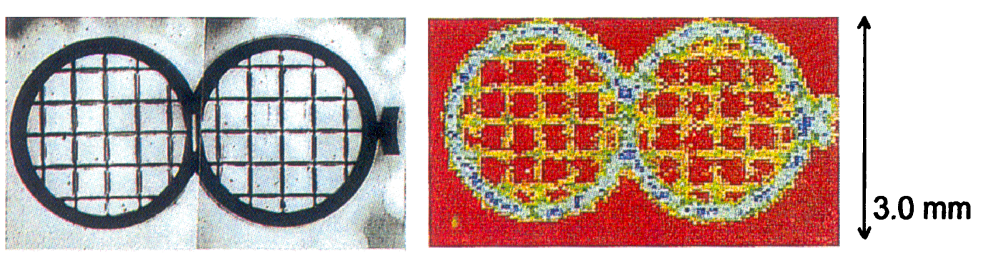

\section{$6.0 \mathrm{~mm}$}

Figure 3 Spatial resolution test for the $x$-ray scanning apparatus.

a. Light microscope image of a TEM specimen grid.

b. X-ray micro-fluorescence map (Au $\mathrm{L} \alpha$ lines) of gold patches on a glass substrate. (See Color Plate 5). 

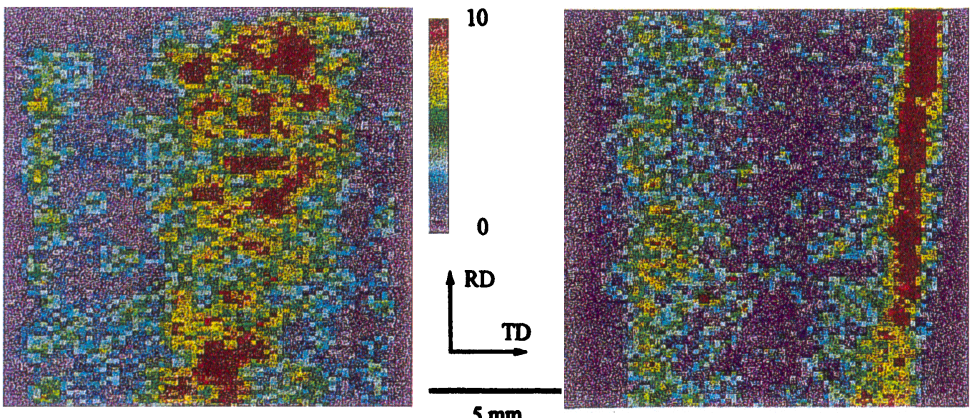

Figure 4 Texture distribution maps of a longitudinal section through a pass rolled copper sample. a. 220 pole in ND, 63.91 image points, $3 \mathrm{sec} /$ point

b. 331 pole in ND, 55.95 image points, 4 sec/point

(See Color Plate 6).

and $\left.\mathrm{E}_{220}=5.7 \mathrm{keV}\right)$. In order to obtain the highest possible intensity in the weaker 331 map (Figure 4.b), the angles were changed to symmetrical diffraction settings $(\theta$ $=\theta_{1}=\theta_{2}=60^{\circ}$ ) such that the diffraction peak was positioned on the characteristic $\mathrm{W} \mathrm{L}_{\alpha}$ line $\left(\mathrm{E}_{331}=8.4 \mathrm{keV}\right)$ of the primary beam. The 220 texture map reveals a wide and rather diffuse strip of high intensity extending from the centre to the right side of the sample. This may be a texture component left over from the initial square profile. The 331 map, however, shows a narrow strip of high intensity on the right and a smaller and less pronouced one on the left hand side. These regions have undergone the highest deformation by the rolling process, and here texture has changed more inhomogeneously.

Figure 5 represents the mip-plane of a partially recrystallised iron profile which had been reduced to $70 \%$ by rolling to $1 \mathrm{~mm}$ thickness. For maximum intensity the Bragg angle was set to $\theta=\theta_{1}=\theta_{2}=31^{\circ}$, in order to enhance intensity by positioning the diffracted Fe 200 peak on the $\mathrm{W} L \alpha$ line $\left(\mathrm{E}_{200}=\mathrm{E}_{\mathrm{w} L \alpha}=8.4 \mathrm{keV}\right)$. Texture is distributed very inhomogeneously in this sample.

The bulk forming process is mainly characterised by the macroscopic parameters distribution of stress, friction between workpiece and forming tool, elastic deformation of workpiece and forming tool, local flux of material, speed and temperature. Finite element methods are now capable to model rather complicated forming processes, and spike forming (Figure 6) is a critical benchmark test for numerical modelling (Schonauer et al., 1993). The integral texture was measured by $\mathrm{x}$-ray diffraction over a cross-section through an aluminium bar in the initial state and after spike forming, Despite the heavy and inhomogeneous deformation of the sample (Figure 6.b), there is no drastic change in microstructure indicated in the integral textures. The recalculated 100, 110 and 111 pole figures as well as the ODF of the initial state (Figure 7) mainly differ in the strength of texture from the pole figures and ODF of the sample after the spike. forming test (Figure 8) rather than in the appearance of new texture components. The deformation by spike forming seems to merely reduce the initial cube texture to about one third in the ODF. However the texture maps reveal pronounced texture inhomogeneities in the cross section (Figure 9.a) and in the longitudinal section (Figure 9.b) after the spike forming test. These inhomogeneities have been produced as supposed by considerably different local deformations. 

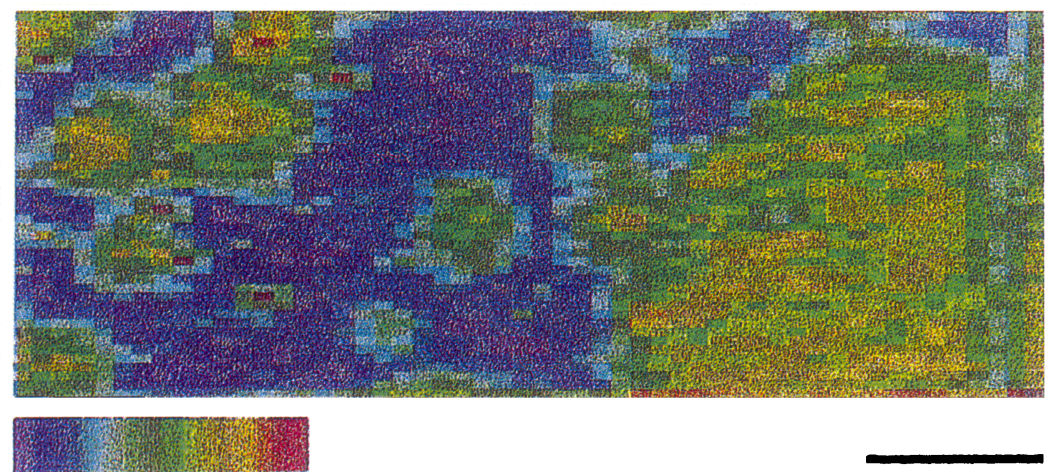

\section{$5 \mathrm{~mm}$}

Figure 5 Texture distribution map of the mid-plane of a partially recrystallised iron sheet (200 pole in normal direction, 55-51 image points, $7 \mathrm{sec} /$ point). (See Color Plate 7).
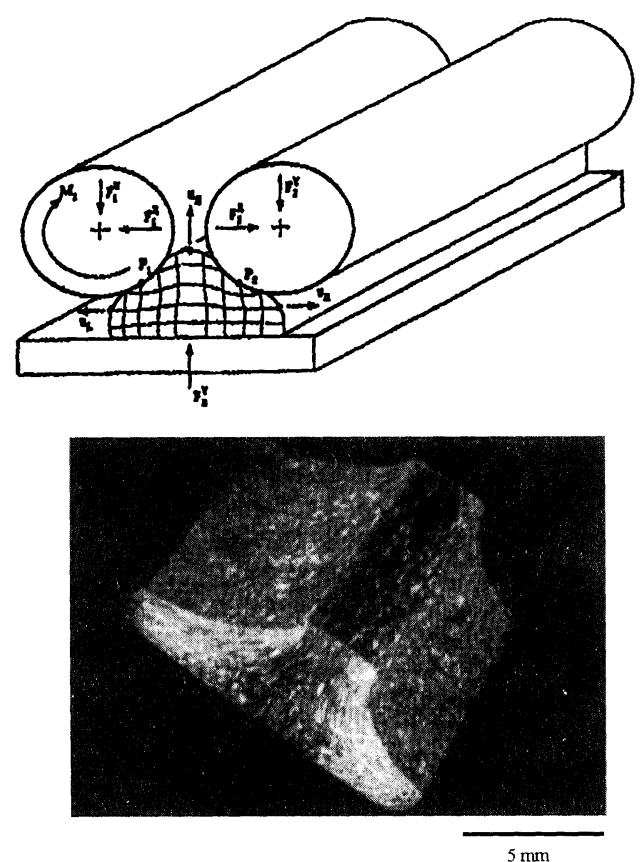

Figure 6 The spike-forming test.

a. Schematics of the experimental set-up.

b. Spike-formed aluminium sample. 

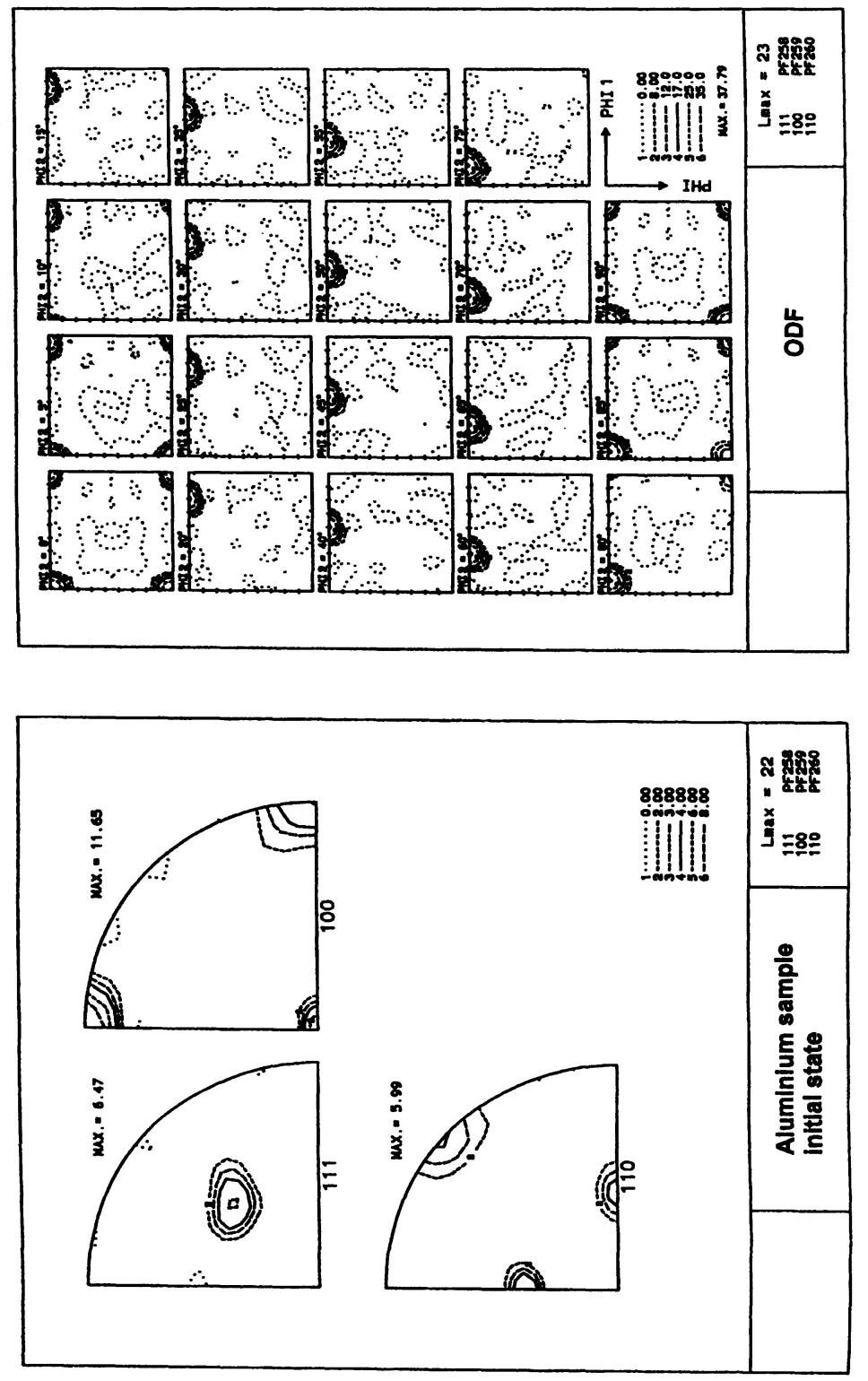

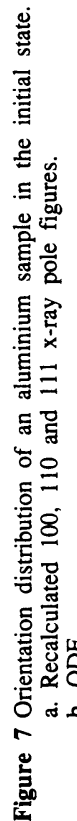



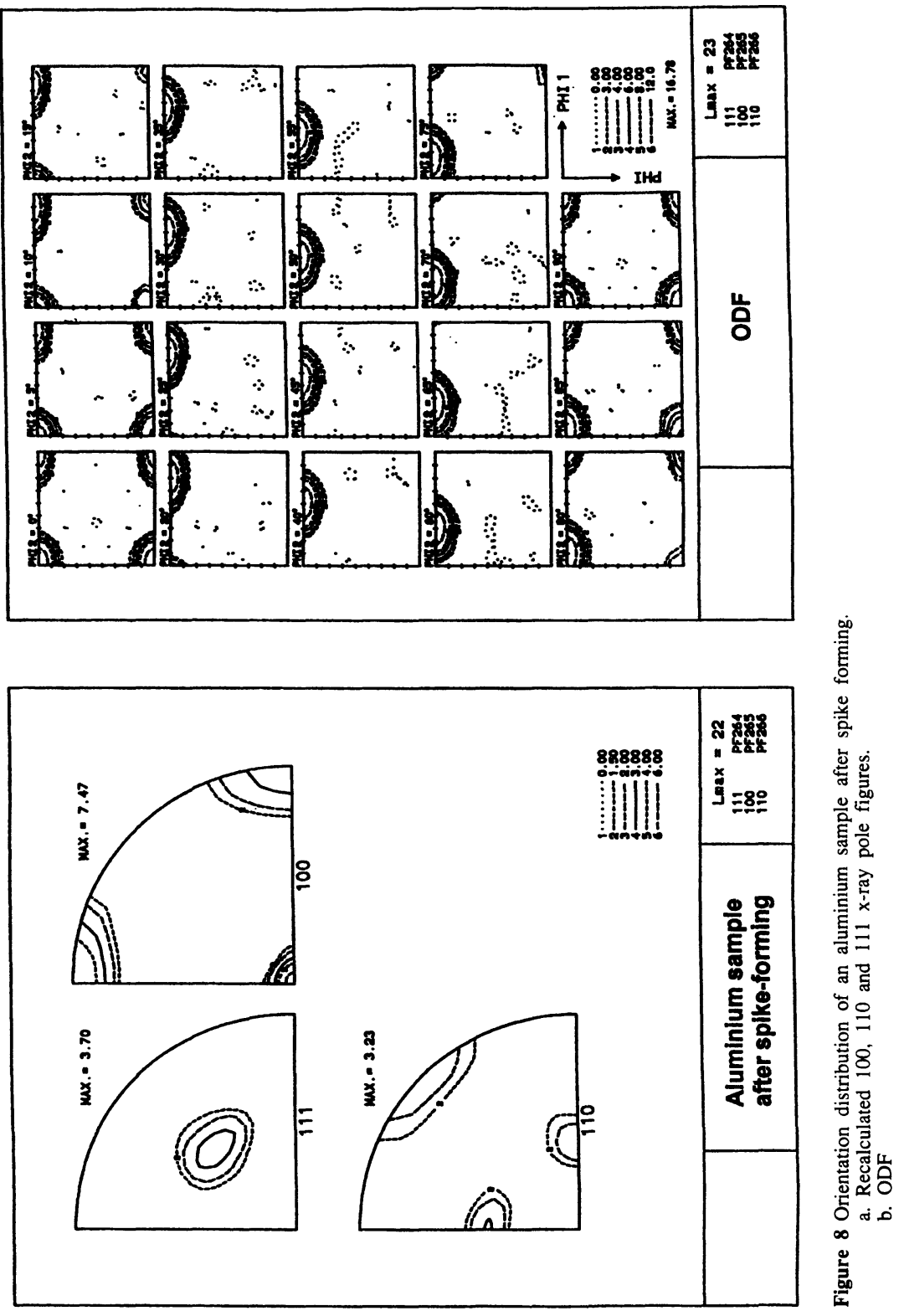

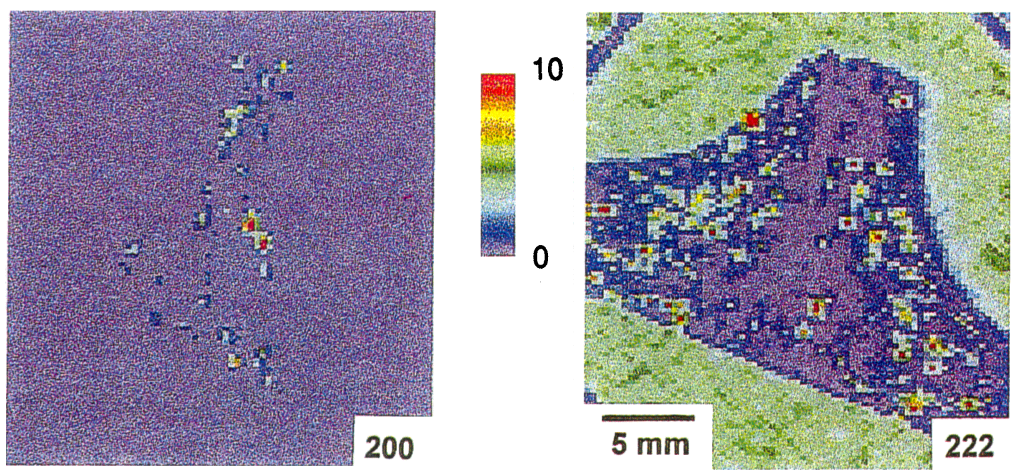

\section{cross section}
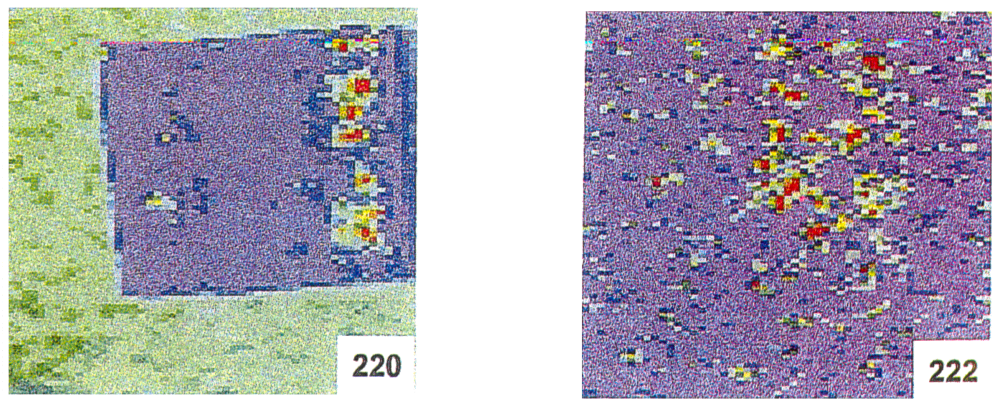

longitudinal section

Figure 9 Texture distribution maps of a spike-formed aluminium sample.

The measured poles are oriented normal to the section plane.

a. Cross section.

b. Longitudinal section.

(See Color Plate 8).

\section{CONCLUSION}

Scanning $x$-ray mapping based on energy dispersive $x$-ray diffraction offers a very powerful technique for texture imaging. Several texture distribution maps can be acquired simultaneously for different hkl reflections but for a common sample direction. All 
reflections are measured at the same Bragg angle setting. It is not necessary to adjust the sample on a Rowland circle. Measurement is not affected by the degree of plastic deformation. In addition to texture mapping, element distributions can be imaged in the same way by analysing characteristic $\mathrm{x}$-ray fluorescence lines. $\mathrm{X}$-ray mapping is a very gentle method, since the specimen is only exposed to $x$-rays and stays in the atmospheric environment. Bulks can be studied irrespective of their conductivity. Due to the large depth of focus with a collimated primary beam, rough surfaces can be imaged. Sample preparation is simple and often a thorough cleaning may just by adequate.

\section{Acknowledgement}

Financial support by the German Research Foundation (DFG Forschergruppe "Textur und Anisotropie kristalliner Stoffe") is gratefully acknowledged.

The authors thank P. Klimanek, BA Freiberg (Germany), for providing the pass-rolled copper sample and T. Rodič, University of Ljubljana (Slovenia), for providing the spikeformed aluminium sample.

\section{References}

Carpenter, D. A., Taylor, M. A. and Holcombe, C. E. (1989). Advances in X-Ray Analysis, 32, 115-120.

Dingley, D. J. and Randle, V. (1992). Journal of Materials Science, 27, 4545-4566.

Helming, K. and Schwarzer, R. A. (1994). Materials Science Forum, 157-162, 1219-1224.

Larsson, S. and Engström, P. (1992). Advances in X-Ray Analysis, 35, 1019-1025.

Mason, T. A. and Adams, B. L. (1994). JOM, 46, 43-45.

Pella, P. A. and Feng, L (1992). Advances in X-Ray Analysis, 35, 1063-1067.

Rindby, A., Engström, P. Larsson, S. and Stocklassa, B. (1989). X-Ray Spectrometry, 18, 109-112.

Schonauer, M., Rodič, T. and Owen, D. R. J. (1993). J. de Physique III 3, 1199-1209.

Schwarzer, R. A. (1989). Proc. 12th Intern. Congr. X-Ray Optics and Microanalysis, Cracow, 205-208.

Schwarzer, R. A. (1991a). Textures and Microstructures, 14-18, 7-27.

Schwarzer, R. A. (1991b). Textures and Microstructures, 14-18, 241-244.

Schwarzer, R. A. (1993). Steel Research, 64, 570-574.

Schwarzer, R. A. and Kunze, K. (1994). Advances in X-Ray Analysis, 38, in print.

Schwarzer, R. A. and Zaefferer, S. (1994). Advances in X-Ray Analysis, 38, in print.

Weiland, H. (1994). JOM, 46, 37-41.

Xia, W. and Schwarzer, R. A. (1994). Materials Science Forum, 157-162, 487-492. 